\title{
Turner's Logic of Universal Causation, Propositional Logic, and Logic Programming
}

\author{
Jianmin $\mathrm{Ji}^{1}$ and Fangzhen $\mathrm{Lin}^{2}$ \\ 1 School of Computer Science and Technology \\ University of Science and Technology of China, Hefei, China \\ jianmin@ustc.edu.cn \\ 2 Department of Computer Science and Engineering \\ The Hong Kong University of Science and Technology, Hong Kong \\ flin@cs.ust.hk
}

\begin{abstract}
Turner's logic of universal causation is a general logic for nonmonotonic reasoning. It has its origin in McCain and Turner's causal action theories which have been translated to propositional logic and logic programming with nested expressions. In this paper, we propose to do the same for Turner's logic, and show thatTurner's logic can actually be mapped to McCain and Turner's causal theories. These results can be used to construct a system for reasoning in Turner's logic.
\end{abstract}

\section{Introduction}

Turner's logic of universal causation [17], called UCL, is a nonmonotonic modal logic that generalizes McCain and Turner's causal action theories [15]. The idea is to use the modal operator $\mathbf{C}$ to specify the statement that a proposition is "caused". For instance, $\psi \supset \mathbf{C} \phi$ says that $\phi$ is caused whenever $\psi$ obtains.

McCain and Turner's causal action theories have been the basis for the semantics of several expressive action languages, such as $\mathcal{C}$ and $\mathcal{C}+[11,5]$. They have been translated to propositional logic and logic programming. Ferraris [2] provided a translation from causal theories to disjunctive logic programs. Lee [9] proposed a conversion from causal theories to propositional logic. In this paper, we consider UCL, and show that UCL theories can be converted to propositional theories. We also show that they can be converted to logic programs with nested expressions in polynomial size with polynomial number of new variables. This result improves and generalizes Turner's linear and modular translation from a fragment of UCL to disjunctive logic programs [17]. Furthermore we show that both Ferraris and Lee's translations are special cases of our translations, just as McCain and Turner's causal theories are special theories in UCL. Our motivation for this work is to use the translations to implement a system for computing UCL theories via SAT solvers or ASP solvers, like the system CCalc ${ }^{3}$ for causal theories.

\footnotetext{
$\overline{{ }^{3} \text { http://www }} \cdot$ cs.utexas.edu/ tag/ccalc/.
} 
This paper is organized as follows. Section 2 reviews UCL and logic programming. Section 3 shows how Turner's logic can be mapped to propositional logic. Section 4 considers mapping UCL theories to logic programs with nested expressions. Section 5 outlines how the translations here are related to Ferraris and Lee's translations. Finally, Section 6 concludes this paper.

\section{Preliminaries}

\subsection{Propositional languages}

We assume a propositional language with two zero-place logical connectives $\top$ for tautology and $\perp$ for contradiction. We denote by Atom the set of atoms, the signature of our language, and Lit the set of literals: Lit $=$ Atom $\cup\{\neg a \mid$ $a \in A$ tom $\}$. A set $I$ of literals is called complete if for each atom $a$, exactly one of $\{a, \neg a\}$ is in $I$. Given a literal $l$, the complement of $l$, written $\bar{l}$ below, is $\neg a$ if $l$ is $a$ and $a$ if $l$ is $\neg a$, where $a$ is an atom. For a set $L$ of literals, we let $\bar{L}=\{\bar{l} \mid l \in L\}$.

In this paper, we identify an interpretation with a complete set of literals. If $I$ is a complete set of literals, we use it as an interpretation when we say that it is a model of a formula, and we use it as a set of literals when we say that it entails a formula.

\subsection{Turner's logic of universal causation}

The language of Turner's logic of universal causation (UCL) [17] is a modal propositional language with a modal operator C. UCL formulas are propositional formulas with unary modal operator C. A UCL theory is a set of UCL formulas.

The semantics of UCL is defined through causally explained interpretations. A UCL structure is a pair $(I, \mathcal{S})$ such that $I$ is an interpretation, and $\mathcal{S}$ is a set of interpretations to which $I$ belongs. The truth of a UCL sentence in a UCL structure is defined by the standard recursions over the propositional connectives, plus the following two conditions:

$$
\begin{gathered}
(I, \mathcal{S}) \models a \text { iff } I \models a \quad(\text { for any atom } a) \\
(I, \mathcal{S}) \models \mathbf{C} \phi \text { iff for all } I^{\prime} \in \mathcal{S},\left(I^{\prime}, \mathcal{S}\right) \models \phi
\end{gathered}
$$

Given a UCL theory $T$, we write $(I, \mathcal{S}) \models T$ to mean that $(I, \mathcal{S}) \models \phi$, for every $\phi \in T$. In this case, we say that $(I, \mathcal{S})$ is a model of $T$. We also say that $(I, \mathcal{S})$ is an $I$-model of $T$, emphasizing the distinguished interpretation $I$.

Let $T$ be a UCL theory. An interpretation $I$ is causally explained by $T$ if $(I,\{I\})$ is the unique $I$-model of $T$.

Note that, if there is a nested occurrence of $\mathbf{C}$, the $\mathbf{C}$ that occurs in the range of another $\mathbf{C}$ can be equivalently ${ }^{4}$ removed [17]. In the paper, we only consider UCL formulas with no nested occurrences of $\mathbf{C}$. A formula of the form $\mathbf{C} \phi$, where $\phi$ is a propositional formula, is called a $\mathbf{C}$-atom. Then these UCL formulas are constructed from $\mathbf{C}$-atoms, propositional atoms and connectives.

\footnotetext{
${ }^{4}$ In the sense that, two formulas have the same set of UCL models.
} 


\subsection{Logic Programming}

A nested expression is built from literals using the 0-place connectives $\top$ and $\perp$, the unary connective "not" and the binary connective "," and ";".

A logic program with nested expressions is a finite set of rules of the form $F \leftarrow G$, where $F$ and $G$ are nested expressions.

The answer set of a logic program with nested expressions is defined as in [12]. Given a nested expression $F$ and a set $S$ of literals, we define when $S$ satisfies $F$, written $S=F$ below, recursively as follows ( $l$ is a literal and $G$ is a nested expression):

$-S=l$ if $l \in S$,

$-S \models \top$ and $S \not \models \perp$,

$-S \models n o t F$ if $S \not \models F$,

$-S \models F, G$ if $S \models F$ and $S \models G$, and

- $S \models F ; G$ if $S \models F$ or $S \models G$.

$S$ satisfies a rule $F \leftarrow G$ if $S \models F$ whenever $S \models G$. $S$ satisfies a logic program $P$, written $S \models P$, if $S$ satisfies all rules in $P$.

The reduct $P^{S}$ of $P$ related to $S$ is the result of replacing every maximal subexpression of $P$ that has the form not $F$ with $\perp$ if $S \models F$, and with $T$ otherwise.

Let $P$ be a logic program without not, the answer set of $P$ is any minimal consistent subset $S$ of $L i t$ that satisfies $P$. We use $\Gamma_{P}(S)$ to denote the set of answer sets of $P^{S}$. Now a consistent set $S$ of literals is an answer set of $P$ iff $S \in \Gamma_{P}(S)$.

Every logic program with nested expressions can be equivalently translated to disjunctive logic programs with disjunctive rules of the form

$$
l_{1} \vee \cdots \vee l_{k} \leftarrow l_{k+1}, \ldots, l_{t}, \text { not } l_{t+1}, \ldots, \text { not } l_{m}, \text { not not } l_{m+1}, \ldots, \text { not not } l_{n} \text {, }
$$

where $n \geq m \geq t \geq k \geq 0$ and $l_{1}, \ldots, l_{n}$ are propositional literals. A disjunctive logic program can be computed by disjunctive ASP solvers such as claspD [1], DLV [10], GNT [7] and cmodels [6].

\section{From Turner's Logic of Universal Causation to Propositional Logic}

Before presenting the translation, we provide some notations. Given a UCL formula $F$, let $\operatorname{Atom}_{\mathbf{C}}(F)=\{\phi \mid \mathbf{C} \phi$ is a $\mathbf{C}$-atom occurring in $F\}$. Given a UCL theory $T$, we let $\operatorname{Atom}_{\mathbf{C}}(T)=\bigcup_{F \in T} \operatorname{Atom}_{\mathbf{C}}(F)$.

We use $\operatorname{tr}_{p}(F)$ to denote the propositional formula obtained from the UCL formula $F$ by replacing each occurrence of a $\mathbf{C}$-atom $\mathbf{C} \phi$ by a new propositional atom $a_{\phi}$ w.r.t. $\phi$.

Given two propositional formulas $\phi$ and $\psi$, we use $\phi^{\psi}$ to denote the propositional formula obtained from $\phi$ by replacing each occurrence of an atom $a$ with a new atom $a^{\psi}$ w.r.t. $\psi$. 
The following proposition provides a specification of the propositional formula whose models are related to models of a UCL theory.

Proposition 1. Let $T$ be a $U C L$ theory. A UCL structure $(I, \mathcal{S})$ is a model of $T$ if and only if there exists a model $I^{*}$ of the propositional formula

$$
\begin{aligned}
\bigwedge_{F \in T} \operatorname{tr}_{p}(F) \wedge & \bigwedge_{\phi \in \operatorname{Atom}_{\mathbf{C}}(T)}\left(a_{\phi} \supset \phi\right) \\
& \wedge \bigwedge_{\psi \in \text { Atom }_{\mathbf{C}}(T)}\left(\left(\neg a_{\psi} \wedge \psi\right) \supset\left(\bigwedge_{\phi \in \operatorname{Atom}_{\mathbf{C}}(T)}\left(a_{\phi} \supset \phi^{\psi}\right) \wedge \neg \psi^{\psi}\right)\right),
\end{aligned}
$$

such that $I^{*} \cap$ Lit $=I$ and for each $\phi \in \operatorname{Atom}_{\mathbf{C}}(T), a_{\phi} \in I^{*}$ iff $\mathcal{S} \models \phi$.

Proof. " $\Rightarrow "(I, \mathcal{S})$ is a model of $T$, then $I \in \mathcal{S}$. If $\mathcal{S} \not \models \psi$ and $I \models \psi$, then there exists another interpretation $I^{\prime} \in \mathcal{S}$ such that $I^{\prime} \models \neg \psi$. Thus, we can create an interpretation $I^{*}$ such that

$$
\begin{gathered}
I^{*}=I \cup\left\{a_{\phi} \mid \phi \in \operatorname{Atom}_{\mathbf{C}}(T) \text { and } \mathcal{S}=\phi\right\} \cup\left\{\neg a_{\phi} \mid \phi \in \operatorname{Atom}_{\mathbf{C}}(T) \text { and } \mathcal{S} \not \models \phi\right\} \\
\cup \bigcup_{\psi \in \text { Atom }_{\mathbf{C}}(T), \mathcal{S} \models \psi}\left\{l^{\psi} \mid l \in I\right\} \cup \bigcup_{\psi \in \text { Atom }_{\mathbf{C}}(T), \mathcal{S} \mid \neq \psi, \exists I^{\prime} . I^{\prime} \in \mathcal{S}, I^{\prime} \models \neg \psi}\left\{l \in I^{\prime}\right\} .
\end{gathered}
$$

Clearly, $I^{*} \models(1)$.

" $\Leftarrow " I^{*} \models T$. Let $I=I^{*} \cap L i t$ and $\mathcal{S}=\left\{I^{\prime} \mid\right.$ if $I^{*} \models a_{\phi}$ for some $\phi \in \operatorname{Atom}_{\mathbf{C}}(T)$, then $I^{\prime}=\phi$ \}. Note that, $I^{*} \models \bigwedge_{\phi \in \operatorname{Atom}_{\mathbf{C}}(T)}\left(a_{\phi} \supset \phi\right)$, then $I \in \mathcal{S}$. For each $\phi \in \operatorname{Atom}_{\mathbf{C}}(T)$, if $I^{*} \models a_{\phi}$, then $\mathcal{S} \models \phi$; from (1), if $I^{*} \models \neg a_{\phi}$, then there exists an interpretation $I^{\prime}$ such that $I^{\prime} \models \neg \psi$ and for each $\psi \in \operatorname{Atom}_{\mathbf{C}}(T), I^{*} \models a_{\psi}$ implies $I^{\prime} \models \psi$, thus $I^{\prime} \in \mathcal{S}$ and $\mathcal{S} \not \models \phi$. Clearly, $(I, \mathcal{S}) \models T$.

Intuitively, the formula

$$
\bigwedge_{\psi \in \operatorname{Atom}_{\mathbf{C}}(T)}\left(\left(\neg a_{\psi} \wedge \psi\right) \supset\left(\bigwedge_{\phi \in \text { Atom }_{\mathbf{C}}(T)}\left(a_{\phi} \supset \phi^{\psi}\right) \wedge \neg \psi^{\psi}\right)\right)
$$

specifies that for each UCL structure $(I, \mathcal{S})$, if $I \models \psi$ and $\mathcal{S} \models \neg \mathbf{C} \psi$, then there exists an interpretation $I^{\prime} \in \mathcal{S}$ such that $I^{\prime}=\neg \psi$.

In the following, we construct propositional formulas whose models are related to causally explained interpretations. First, we consider how to specify the unique model of a propositional formula.

Given a propositional formula $\phi$ and a nonempty consistent set $K$ of literals, we denote by $\left.\phi\right|_{K \rightarrow \perp}$ the result of replacing each occurrence of an atom $a$ in $\phi$ by $\perp$ if $a \in K$ and $\top$ if $\neg a \in K$.

Lemma 1. Let $\phi$ be a propositional formula, $K$ a nonempty consistent set of literals, and an interpretation $I \supseteq K .\left.I \not \models \bigwedge_{l \in K} l \supset \neg \phi\right|_{K \rightarrow \perp}$ if and only if the interpretation $(I \backslash K) \cup \bar{K} \models \phi$. 
Proof. Let $I^{\prime}=(I \backslash K) \cup \bar{K}$.

" $\left.\Rightarrow " I \not \models \bigwedge_{l \in K} l \supset \neg \phi\right|_{K \rightarrow \perp}$, then $\left.I \models \phi\right|_{K \rightarrow \perp}$. Note that, atoms occurring in $K$ do not occur in $\left.\phi\right|_{K \rightarrow \perp}$, then $\left.I^{\prime} \models \phi\right|_{K \rightarrow \perp}$, furthermore, $I^{\prime}=\bar{K}$, thus $I^{\prime} \models \phi$.

" $\Leftarrow " I^{\prime}=\phi$ and $I^{\prime}=\bar{K}$, then $\left.I^{\prime} \models \phi\right|_{K \rightarrow \perp}$, thus $\left.I \models \phi\right|_{K \rightarrow \perp}$. Note that $K \subseteq I$, then $\left.I \not \forall \bigwedge_{l \in K} l \supset \neg \phi\right|_{K \rightarrow \perp}$.

To avoid influence of auxiliary atoms, we introduce the notion of forgetting provided by Lin and Reiter [14].

Definition 1. Let $\phi$ be a propositional formula and $S$ a set of atoms. forget $(\phi ; S)$ is the formula inductively defined as follows:

$-\operatorname{forget}(\phi ; \emptyset)=\phi$,

$-\operatorname{forget}(\phi ;\{a\})=\left.\left.\phi\right|_{\{a\} \rightarrow \perp} \vee \phi\right|_{\{\neg a\} \rightarrow \perp}$,

- forget $(\phi ;\{a\} \cup S)=\operatorname{forget}($ forget $(\phi ; S),\{a\})$.

Lemma 2 (Theorem 4 in [14]). Let $\phi$ be a propositional formula and $S$ a set of atoms. An interpretation $I \models$ forget $(\phi ; S)$ if and only if there exists an interpretation $I^{\prime} \models \phi$ such that $I \backslash S \cup \bar{S}=I^{\prime} \backslash S \cup \bar{S}$.

Directly from Lemma 1 and 2, we have the following lemma.

Lemma 3. Let $\phi$ be a propositional formula, $K$ a nonempty consistent set of literals, $S$ a set of atoms, and an interpretation $I \supseteq K . I \forall \bigwedge_{l \in K} l \supset$ $\neg$ forget $\left.(\phi ; S)\right|_{K \rightarrow \perp}$ if and only if there exists an interpretation $I^{\prime} \models \phi$ such that $((I \backslash S \cup \bar{S}) \backslash K) \cup \bar{K}=I^{\prime} \backslash S \cup \bar{S}$.

Given a propositional formula $\phi$, we use $\widehat{\phi}$ to denote the propositional formula obtained from $\phi$ by replacing each occurrence of an atom $a$ in $\phi$ by a new atom $\hat{a}$. For a set $L$ of literals, we let $\widehat{L}=\{\hat{l} \mid l \in L\}$. We use $L i t_{a}$ to denote the set of literals formed from new atoms of the form $a_{\phi}$ and Atom* ${ }^{*}$ the set of atoms of the form $a^{\psi}$ w.r.t. $\psi$ in (1).

Theorem 1. Let $T$ be a UCL theory. An interpretation I is causally explained by $T$ if and only if there exists a model $I^{*}$ of the propositional formula

$$
\begin{gathered}
(1) \wedge \bigwedge_{\phi \in \operatorname{Atom}_{\mathbf{C}}(T)}\left(a_{\phi} \supset \widehat{\phi}\right) \\
\wedge \bigwedge_{\substack{A \subseteq L i t_{a} \\
A \text { is nonempty and consistent }}}\left(\left.\bigwedge_{l_{a} \in A} l_{a} \supset \neg \operatorname{forget}\left((1) ; \text { Atom }^{*}\right)\right|_{A \rightarrow \perp}\right) \\
\wedge \bigwedge_{K \text { is nonempty and consistent }}\left(\left.\bigwedge_{l \in K} \hat{l} \supset \neg \bigwedge_{\phi \in \operatorname{Atom}_{\mathbf{C}}(T)}\left(a_{\phi} \supset \widehat{\phi}\right)\right|_{\widehat{K} \rightarrow \perp}\right),
\end{gathered}
$$

such that $I^{*} \cap$ Lit $=I$. 
Proof. " $\Rightarrow " I$ is causally explained by $T$, then $(I,\{I\})$ is the unique $I$-model of $T$. We can create an interpretation $I^{*}$ such that

$$
\begin{array}{r}
I^{*}=I \cup\left\{a_{\phi} \mid \phi \in \operatorname{Atom}_{\mathbf{C}}(T) \text { and } I \models \phi\right\} \cup\left\{\neg a_{\phi} \mid \phi \in \operatorname{Atom}_{\mathbf{C}}(T) \text { and } I \not \models \phi\right\} \\
\cup \bigcup_{\psi \in \operatorname{Atom}_{\mathbf{C}}(T)}\left\{l^{\psi} \mid l \in I\right\} \cup\{\hat{l} \mid l \in I\} .
\end{array}
$$

From Proposition $1, I^{*} \models(1) \wedge \bigwedge_{\phi \in \text { Atom }_{\mathbf{C}}(T)}\left(a_{\phi} \supset \widehat{\phi}\right)$.

If $I^{*} \forall \bigwedge_{l_{a} \in A} l_{a} \supset \neg$ forget $\left.\left((1) ;\right.$ Atom $\left.^{*}\right)\right|_{A \rightarrow \perp}$ for some nonempty consistent set $A \subseteq$ Lit $_{a}$, similar to the proof of Lemma 3 , then these exists another interpretation $I^{* \prime}$ such that $\left(\left(I^{*} \backslash\right.\right.$ Atom $\left.\left.{ }^{*} \cup \overline{\text { Atom }^{*}}\right) \backslash A\right) \cup \bar{A}=I^{* \prime} \backslash$ Atom $^{*} \cup \overline{\text { Atom }^{*}}$ and $I^{* \prime} \models(1)$. From Proposition 1, there exists another set $\mathcal{S}^{\prime}$ of interpretations such that $\mathcal{S}^{\prime} \neq\{I\}, I \in \mathcal{S}^{\prime}$ and $\left(I, \mathcal{S}^{\prime}\right)$ is an $I$-model of $T$, which conflicts to the condition that $(I,\{I\})$ is the unique $I$-model of $T$.

If $\left.I^{*} \forall \bigwedge_{l \in K} \hat{l} \supset \neg \bigwedge_{\phi \in \text { Atom }_{\mathbf{C}}(T)}\left(a_{\phi} \supset \widehat{\phi}\right)\right|_{\widehat{K} \rightarrow \perp}$ for some nonempty consistent set $K \subseteq$ Lit, similar to the proof of Lemma 1, then there exists another interpretation $I^{* \prime}$ such that $I^{* \prime}=\left(I^{*} \backslash \widehat{K}\right) \cup \overline{\widehat{K}}$ and $I^{* \prime} \models(1) \wedge \bigwedge_{\phi \in \operatorname{Atom}_{\mathbf{C}}(T)}\left(a_{\phi} \supset \widehat{\phi}\right)$. From Proposition 1, there exists another interpretation $I^{\prime}$ such that $\left(I,\left\{I, I^{\prime}\right\}\right) \models$ $T$, which conflicts to the condition that $(I,\{I\})$ is the unique $I$-model of $T$, thus $I^{*} \models(2)$.

"६" $I^{*} \models(2)$. Let $I=I^{*} \cap$ Lit, if there exists another UCL structure $(I, \mathcal{S})$ such that $(I, \mathcal{S}) \models T$ and $\mathcal{S} \neq\{I\}$, then there are two cases: 1. there exists $\phi \in \operatorname{Atom}_{\mathbf{C}}(T)$ such that $I \models \phi$ and $\mathcal{S} \not \models \phi ; 2$. for each $\phi \in \operatorname{Atom}_{\mathbf{C}}(T), I \models \phi$ if and only if $\mathcal{S}=\phi$.

For case 1 , let $A=\left\{a_{\phi} \mid \phi \in \operatorname{Atom}_{\mathbf{C}}(T), I \models \phi, \mathcal{S} \not \models \phi\right\}$, then $I^{*} \models \bigwedge_{l_{a} \in A} l_{a}$ and $\left.I^{*} \models \operatorname{forget}\left((1) ;\right.$ Atom $\left.^{*}\right)\right|_{A \rightarrow \perp}$, which conflicts to the condition that $I^{*} \models$ (2), thus it is impossible.

For case 2, let $I^{\prime} \in \mathcal{S}$ and $I^{\prime} \neq I$, then for each $\phi \in \operatorname{Atom}_{\mathbf{C}}(T), I^{*} \models a_{\phi}$ implies $I^{\prime} \models \phi$, thus there exists $K=I \backslash I^{\prime}$ such that $I^{*} \models \bigwedge_{l \in K} \hat{l}$ and $I^{*} \models$ $\left.\bigwedge_{\phi \in \operatorname{Atom}_{\mathbf{C}}(T)}\left(a_{\phi} \supset \widehat{\phi}\right)\right|_{\widehat{K} \rightarrow \perp}$, which conflicts to the condition that $I^{*} \models(2)$. So $I$ is the only interpretation that satisfies $\left\{\phi \in \operatorname{Atom}_{\mathbf{C}}(T) \mid I^{*} \models a_{\phi}\right\}$, then $(I,\{I\})$ is the unique $I$-model of $T$.

Note that, the size of formula (2) is exponential increased from $T$, as the number of all possible nonempty consistent sets of literals is $3^{n}$, where $n$ is the number of atoms. In fact, we only need to consider a subset of these sets. Details are proposed in Section 5.3.

As a simple example, given the UCL theory $T=\{\mathbf{C}(p \vee q), \mathbf{C} p \supset \mathbf{C} q, \mathbf{C} q \supset$ $\mathbf{C} p$ \}, from the definition of (1), we obtain the following propositional formula:

$$
\begin{aligned}
a_{p \vee q} \wedge\left(a_{p} \equiv a_{q}\right) \wedge & (p \vee q) \wedge\left(a_{p} \supset p\right) \wedge\left(a_{q} \supset q\right) \wedge \\
& \left(\left(\neg a_{p} \wedge p\right) \supset\left(\neg p^{2} \wedge q^{2}\right)\right) \wedge\left(\left(\neg a_{q} \wedge q\right) \supset\left(\neg q^{3} \wedge p^{3}\right)\right)
\end{aligned}
$$


From the definition of (2), we obtain the following formula ${ }^{5}$

$$
\begin{aligned}
(3) \wedge\left(a_{p \vee q} \supset(\hat{p} \vee \hat{q})\right) \wedge\left(a_{p} \supset \hat{p}\right) \wedge\left(a_{q} \supset \hat{q}\right) \\
\wedge\left(a_{p} \supset \neg\left(\neg a_{q}\right)\right) \wedge\left(a_{q} \supset \neg\left(\neg a_{p}\right)\right) \wedge\left(\left(a_{p} \wedge a_{q}\right) \supset \perp\right) \\
\wedge\left(\neg a_{p} \supset \neg\left(a_{q} \wedge p \wedge q\right)\right) \wedge\left(\neg a_{q} \supset \neg\left(a_{p} \wedge p \wedge q\right)\right) \wedge\left(\neg a_{p} \wedge \neg a_{q} \supset \neg(p \wedge q)\right) \\
\wedge\left(\hat{p} \supset \neg\left(\left(a_{p \vee q} \supset \hat{q}\right) \wedge \neg a_{p} \wedge\left(a_{q} \supset \hat{q}\right)\right)\right) \wedge\left(\hat{q} \supset \neg\left(\left(a_{p \vee q} \supset \hat{p}\right) \wedge\left(a_{p} \supset \hat{p}\right) \wedge \neg a_{q}\right)\right) \\
\wedge\left(\neg \hat{p} \supset \neg\left(a_{q} \supset \hat{q}\right)\right) \wedge\left(\neg \hat{q} \supset \neg\left(a_{p} \supset \hat{p}\right)\right)
\end{aligned}
$$

where $\hat{p}$ and $\hat{q}$ are new atoms. The formula implies that

$$
a_{p \vee q} \wedge \hat{p} \wedge \hat{q} \wedge a_{p} \wedge a_{q} \wedge p \wedge q \wedge\left(\left(a_{p} \wedge a_{q}\right) \supset \perp\right)
$$

which is inconsistent. From Theorem 1, there does not exist an interpretation $I$ such that $I$ is causally explained by the UCL theory $T$.

\section{From Turner's Logic of Universal Causation to Logic Programming}

Formula (2) in propositional logic is complex, as it needs to include constraints to make it satisfied by a "unique model". The problem becomes easier when we consider logic programming. Based on the propositional formula (1), we can translate a UCL theory $T$ to a logic program with nested expressions.

Note that, every propositional formula $\phi$ can be equivalently translated to $\mathrm{CNF}$ as

$$
\left(l_{1}^{1} \vee \cdots \vee l_{n^{1}}^{1}\right) \wedge \cdots \wedge\left(l_{1}^{m} \vee \cdots \vee l_{n^{m}}^{m}\right),
$$

where $l_{1}^{1}, \ldots, l_{n^{m}}^{m}$ are literals.

For any propositional formula, we can convert it to the nested expression by replacing each $\wedge$ with a comma, each $\vee$ with a semicolon and $\neg$ with not.

Given a UCL theory $T$, we use $\operatorname{tr}_{n e}(T)$ to denote the nested expression obtained from (1). We use Atom' to denote the set of atoms that occur in (1) but not in Atom. Now we define $\operatorname{tr}_{l p}(T)$ to be the logic program containing $\perp \leftarrow \operatorname{nottr}_{n e}(T)$, the following rules for each $\phi \in \operatorname{Atom}_{\mathbf{C}}(T)$ whose CNF is in the form of (4)

$$
\begin{aligned}
l_{1}^{1} ; \ldots ; l_{n^{1}}^{1} & \leftarrow \operatorname{not} \operatorname{not} a_{\phi},\left(\bar{l}_{1}^{1} ; \operatorname{not} \bar{l}_{1}^{1}\right), \ldots,\left(\bar{l}_{n^{1}}^{1} ; \operatorname{not} \bar{l}_{n^{1}}^{1}\right), \\
& \ldots \\
l_{1}^{m} ; \ldots ; l_{n^{m}}^{m} & \leftarrow \operatorname{not} \operatorname{not} a_{\phi},\left(\bar{l}_{1}^{m} ; \operatorname{not} \bar{l}_{1}^{m}\right), \ldots,\left(\bar{l}_{n^{m}}^{m} ; \operatorname{not} \bar{l}_{n^{m}}^{m}\right),
\end{aligned}
$$

and

$$
a^{\prime} ; \neg a^{\prime} \leftarrow \top, \quad \text { (for each } a^{\prime} \in \text { Atom }^{\prime} \text { ). }
$$

\footnotetext{
$\overline{5}$ The formula is simplified due to Theorem 5 in Section 5.3.
} 
Lemma 4. Let $T$ be a UCL theory and $I$ and $J$ two interpretations. $(I,\{I, J\}) \models$ $T$ if and only if there exists a set $S$ of literals occurring in $\operatorname{tr}_{l p}(T)$ such that $S \models\left(\operatorname{tr}_{l p}(T)\right)^{S \cup I}$ and $S \cap L i t=I \cap J$.

Proof. " $\Rightarrow$ " $(I,\{I, J\}) \models T$. Similar to the proof of Proposition 1, we can create an interpretation $I^{*}$ such that $I^{*} \cap L i t=I$ and $I^{*}=(1)$. Note that, $\left(\operatorname{tr}_{l p}(T)\right)^{I^{*}}$ contains rules of the form

$$
l_{1} ; \ldots ; l_{n} \leftarrow \underset{l \in\left\{l_{1}, \ldots, l_{n}\right\}, \bar{l} \in I}{ } \bar{l},
$$

where $I^{*} \models a_{\phi}$ for corresponding $\phi \in \operatorname{Atom}_{\mathbf{C}}(T)$.

Note that, $\{I, J\} \models \phi, I \models l_{1} \vee \cdots \vee l_{n}$ and $J \models l_{1} \vee \cdots \vee l_{n}$. Consider the case, for each literal $l \in\left\{l_{1}, \ldots, l_{n}\right\}, \bar{l} \in I$ implies $\bar{l} \in I \cap J$, then there exists literal $l \in\left\{l_{1}, \ldots, l_{n}\right\}$ and $l \in J$ such that $l \in I$ (if not, $\bar{l} \in I$ which implies $\bar{l} \in J)$, thus $(I \cap J) \models(5)$.

We denote $S=\left(I^{*} \backslash I\right) \cup(I \cap J)$. Clearly, $S=\left(\operatorname{tr}_{l p}(T)\right)^{S \cup I}$.

" $\Leftarrow " S=\left(\operatorname{tr}_{l p}(T)\right)^{S \cup I}$ and $S \cap L i t=I \cap J .\left(\operatorname{tr}_{l p}(T)\right)^{S \cup I}$ contains rules of (5) and $S \models a_{\phi}$ for corresponding $\phi \in \operatorname{Atom}_{\mathbf{C}}(T)$.

Note that $I \cap J \models(5)$, then $I \cap J \models l_{1} \vee \cdots \vee l_{n}$ whenever $\bar{l} \in I \cap J$ for all $\bar{l} \in I$ and $l \in\left\{l_{1}, \ldots, l_{n}\right\}$. If $J \not \models l_{1} \vee \cdots \vee l_{n}$, then there exists $\bar{l} \in I$ and $\bar{l} \notin I \cap J$, thus $\bar{l} \notin J$ and $l \in J$ which conflicts to $J \not \models l_{1} \vee \cdots \vee l_{n}$. So $J \models \phi$, from Proposition $1,(I,\{I, J\}) \models T$.

Theorem 2. Let $T$ be a UCL theory. An interpretation I is causally explained by $T$ if and only if there exists an answer set $S$ of the logic program $\operatorname{tr}_{l p}(T) \cup\{\perp \leftarrow$ not $a$, not $\neg a \mid a \in$ Atom $\}$, such that $S \cap$ Lit $=I$.

Proof. $I$ is causally explained by $T$ means that $(I,\{I\})$ is the unique $I$-model of $T$. From Lemma 4, this is equivalent to the condition, for every set $S$ of literals occurring in $\operatorname{tr}_{l p}(T)$ and interpretation $J$ such that $S \models\left(\operatorname{tr}_{l p}(T)\right)^{S \cup I}$, $S \cap$ Lit $=I \cap J$ iff $J=I$. This means that there exists an answer set $S$ of $\operatorname{tr}_{l p}(T) \cup\{\perp \leftarrow$ not $a$, not $\neg a \mid a \in$ Atom $\}$ such that $S \cap$ Lit $=I$.

\section{Related Work}

\subsection{Turner's Conversion from a Fragment of UCL to Disjunctive Logic Programming}

Turner [17] proposed a simple translation from a subset of UCL theories to disjunctive logic programs [3] via disjunctive default logic [4].

Turner's translation considers the UCL formula of the form

$$
\mathbf{C}\left(l_{1} \wedge \cdots \wedge l_{k}\right) \wedge l_{k+1} \wedge \cdots \wedge l_{m} \supset \mathbf{C} l_{m+1} \vee \cdots \mathbf{C} l_{n},
$$

where $l_{1}, \ldots, l_{n}$ are literals.

A UCL formula of the form (6) is translated to the disjunctive rule

$$
l_{m+1} \vee \cdots \vee l_{n} \leftarrow l_{1}, \ldots, l_{k}, \text { not } \bar{l}_{k+1}, \ldots, n o t \bar{l}_{m} .
$$


It has been proved that, given a set $T$ of UCL formulas in the form (6), an interpretation $I$ is an answer set of the corresponding disjunctive logic program if and only if $I$ is causally explained by $T$.

When every formula in range of $\mathbf{C}$ is a literal, our translation seems more complex than Turner's translation. However, some steps in the translation can also be simplified. Consider the following proposition proposed in [2].

Proposition 2 (Proposition 1 in [2]). For any literal $l$ and any nested expression $F$, the one-rule logic program

$$
l \leftarrow F,(\bar{l} ; \text { not } \bar{l})
$$

is strongly equivalent to $l \leftarrow F$.

\subsection{Ferraris's Translation from Causal Theories to Logic Programs}

Ferraris [2] proposed a translation from McCain and Turner's causal theories [15] to logic programs with nested expressions. As causal theories can be easily converted into UCL, we show that Ferraris's translation is a special case of our translation proposed in Section 4. First, we briefly review causal theories and Ferraris's translation, then we consider the relation to our translation.

A causal theory according to McCain and Turner [15] is a set of causal laws of the following form

$$
\psi \Rightarrow \phi,
$$

where $\phi$ and $\psi$ are propositional formulas.

Ferraris's translation converts the causal law

$$
\psi \Rightarrow l_{1} \vee \cdots \vee l_{n}
$$

to the rule

$$
l_{1} ; \ldots ; l_{n} \leftarrow \operatorname{not} \operatorname{not} \psi^{\text {ne }},\left(\bar{l}_{1} ; \operatorname{not} \bar{l}_{1}\right), \ldots,\left(\bar{l}_{n} ; \operatorname{not} \bar{l}_{n}\right),
$$

where $\psi^{\text {ne }}$ stands for the nested expression of $\psi$. Theorem 1 in [2] proved that models of a set of causal laws in the form (8) are identical to complete answer sets of the corresponding logic programs.

According to Turner [17], a causal law of the form (7) can be translated to his logic as

$$
\psi \supset \mathbf{C} \phi .
$$

Thus given our translation from Turner's logic to logic programming, we have a translation from McCain and Turner's causal theory to logic programming as well.

A UCL formula of the form (9) is called regular. A regular UCL theory is a set of regular UCL formulas.

Note that, when $T$ is a regular UCL theory, formula (1) in Proposition 1 can be simplified to

$$
\bigwedge_{F \in T} \operatorname{tr}_{p}(F) \wedge \bigwedge_{\phi \in \operatorname{Atom}_{\mathbf{C}}(T)}\left(a_{\phi} \supset \phi\right)
$$


Proposition 3. Let $T$ be a regular $U C L$ theory. A $U C L$ structure $(I, \mathcal{S})$ is a model of $T$ if and only if there exists a model $I^{*}$ of formula (10) such that $I^{*} \cap$ Lit $=I$ and for each $\phi \in \operatorname{Atom}_{\mathbf{C}}(T), a_{\phi} \in I^{*}$ iff $\mathcal{S}=\phi$.

Based on Proposition 3, the translation in Section 4 can also be simplified. Given a regular UCL theory $T$, we use $t r_{n e}^{\prime}(T)$ to denote the nested expression obtained from (10). We define $t r_{l p}^{\prime}(T)$ the same as $t r_{l p}(T)$ except $t r_{n e}(T)$ is replaced by $t r_{n e}^{\prime}(T)$.

Theorem 3. Let $T$ be a regular UCL theory. An interpretation I is causally explained by $T$ if and only if there exists an answer set $S$ of the logic program $\operatorname{tr}_{l p}^{\prime}(T) \cup\{\perp \leftarrow$ not a, not $\neg a \mid a \in$ Atom $\}$, such that $S \cap$ Lit $=I$.

It is easy to find out that, for regular UCL theory $T, \operatorname{tr}_{l p}^{\prime}(T)$ is equivalent to the result of Ferraris's translation.

Our translation in Section 4 can also be specified by Ferraris's translation. First, a UCL theory can be converted to a regular UCL theory.

Theorem 4. Let $T$ be a UCL theory. An interpretation I is causally explained by $T$ if and only if $I$ is causally explained by the regular UCL theory with following formulas

$$
\begin{aligned}
& (1), \\
& a_{\phi} \supset \mathbf{C} \phi, \\
& a^{\prime} \supset \mathbf{C} a^{\prime}, \neg a^{\prime} \supset \mathbf{C} \neg a^{\prime} .
\end{aligned}
$$$$
\left(\text { for each } \phi \in \text { Atom }_{\mathbf{C}}(T)\right. \text { ) }
$$$$
\left(\text { for each } a^{\prime} \in \text { Atom }^{\prime}\right)
$$

Then we can use Ferraris's translation turning the regular UCL theory in the above theorem to a logic program with nested expressions.

\subsection{Lee's Translation from Causal Theories to Propositional Theories with Loop Formulas}

Lee [9] proposed a translation from McCain and Turner's causal theories to propositional theories with loop formulas. In this section, we show that we can also define so called "loop formulas" for our translation in Section 3 and Lee's translation would be a special case.

Given a set $\Pi$ of propositional clauses, i.e. disjunctions of literals, the dependency graph of $\Pi$ is the directed graph $G_{\Pi}$ such that

- the vertices of $G_{\Pi}$ are literals in $\Pi$, and

- for any two vertices $l_{1}, l_{2}$, there is an edge from $l_{1}$ to $l_{2}$ if there is a clause $C \in \Pi$ such that $l_{1}$ and $\overline{l_{2}}$ are in $C$.

A nonempty consistent set $L$ of literals is called a loop of $\Pi$ if for any literals $l_{1}$ and $l_{2}$ in $L$, there is a path from $l_{1}$ to $l_{2}$ in $G_{\Pi}$ such that all the vertices in the path are in $L$, i.e. the $L$-induced subgraph of $G_{\Pi}$ is strongly connected. Specially, the singleton set $\{l\}$ for every literal $l \in$ Lit is a loop. We use $\operatorname{Loop}(\Pi)$ to denote the set of all loops of $\Pi$. 
The loop formula associated with a loop $L$ under a set $\Pi$ of propositional clauses, denoted by $L F(\Pi, L)$, is a sentence of the form:

$$
\left.\bigwedge_{l \in L} l \supset \neg \bigwedge_{C \in \Pi} C\right|_{L \rightarrow \perp}
$$

We can simplify the translation from UCL to propositional logic by loops.

Proposition 4. Let $\Pi$ be a set of propositional clauses,

$$
\bigwedge_{C \in \Pi} C \wedge \bigwedge_{L \in \operatorname{Loop}(\Pi)} L F(\Pi, L) \supset \bigwedge_{C \in \Pi} C \wedge \bigwedge_{\begin{array}{c}
K \subseteq L i t \\
\text { is nonempty } \\
\text { and consistent }
\end{array}}\left(\left.\bigwedge_{l \in K} l \supset \neg \bigwedge_{C \in \Pi} C\right|_{K \rightarrow \perp}\right) .
$$

Proof. Let $L \in \operatorname{Loop}(\Pi), K$ a nonempty consistent set of literals s.t. $L \subseteq K$, and there does not exist an edge of $G_{\Pi}$ from a literal in $L$ to a literal in $K \backslash L$.

There does not exist an edge of $G_{\Pi}$ from a literal in $L$ to a literal in $K \backslash L$, then there does not exist a clause $C$ in $\Pi$ of the form

$$
l_{1} \vee \cdots \vee l_{n}
$$

such that $l_{i} \in L$ and $l_{j} \in K \backslash L$ for some $1 \leq i, j \leq n$. Thus, if $C \in \Pi$ and $C \cap L \neq \emptyset$, then $C \cap(K \backslash L)=\emptyset$.

For each clause $C \in \Pi$, as $L \subseteq K$, there are three different cases.

Case $1, L \cap C=\emptyset$. If $L \cap \bar{C} \neq \emptyset$, then $K \cap \bar{C} \neq \emptyset$, thus $\left.\left.\neg C\right|_{L \rightarrow \perp} \equiv \neg C\right|_{K \rightarrow \perp} \equiv$ $\perp$. If $L \cap \bar{C}=\emptyset$, then $\left.\neg C\right|_{L \rightarrow \perp} \equiv \neg C$, thus $C \wedge L F(\{C\}, L) \supset C \wedge\left(\bigwedge_{l \in K} l \supset\right.$ $\left.\left.\neg C\right|_{K \rightarrow \perp}\right)$.

Case 2, $L \cap C \neq \emptyset, L \cap \bar{C}=\emptyset$, and $K \cap \bar{C}=\emptyset$. From the above condition, $C \cap(K \backslash L)=\emptyset$, then $\left.\left.\neg C\right|_{L \rightarrow \perp} \equiv \neg C\right|_{K \rightarrow \perp}$.

Case $3, K \cap \bar{C} \neq \emptyset$. Then $\left.\neg C\right|_{K \rightarrow \perp} \equiv \perp$, thus $C \wedge L F(\{C\}, L) \supset C \wedge\left(\wedge_{l \in K} l \supset\right.$ $\left.\left.\neg C\right|_{K \rightarrow \perp}\right)$.

Based on the above results,

$$
\bigwedge_{C \in \Pi} C \wedge L F(\Pi, L) \supset \bigwedge_{C \in \Pi} C \wedge\left(\left.\bigwedge_{l \in K} l \supset \neg \bigwedge_{C \in \Pi} C\right|_{K \rightarrow \perp}\right) .
$$

In addition, for every nonempty consistent set $K$ of literals, there always exists a loop $L \subseteq K$ such that there does not exist an edge of $G_{\Pi}$ from a formula in $L$ to a formula in $K \backslash L$. So the proposition is proved.

Given a UCL theory $T$, with a slight abuse of notations, we use $\operatorname{Loop}(T)$ to the set of loops of the set of clauses which are in $\mathrm{CNF}$ of $\phi \in \operatorname{Atom}_{\mathbf{C}}(T)$. Similarly, we use $\operatorname{Loop}_{a}(T)$ to the set of loops of the set of clauses which are in CNF of (1).

Theorem 5. Let T be a UCL theory. An interpretation $I$ is causally explained by $T$ if and only if there exists a model $I^{*}$ of the propositional formula 


$$
\begin{aligned}
(1) \wedge \bigwedge_{\phi \in \operatorname{Atom}_{\mathbf{C}}(T)}\left(a_{\phi} \supset \widehat{\phi}\right) & \\
\wedge \bigwedge_{A \subseteq L i t_{a},}, A \in \operatorname{Loop}_{a}(T) & \left(\left.\bigwedge_{l_{a} \in A} l_{a} \supset \neg \operatorname{forget}\left((1) ; \operatorname{Atom}^{*}\right)\right|_{A \rightarrow \perp}\right) \\
& \wedge \bigwedge_{L \in \operatorname{Loop}(T)}\left(\left.\bigwedge_{l \in L} \hat{l} \supset \neg \bigwedge_{\phi \in \operatorname{Atom}_{\mathbf{C}}(T)}\left(a_{\phi} \supset \widehat{\phi}\right)\right|_{\widehat{L} \rightarrow \perp}\right),
\end{aligned}
$$

such that $I^{*} \cap$ Lit $=I$.

Similar to the discussion in the previous section, when $T$ is a regular UCL theory, the above theorem can be simplified.

Theorem 6. Let $T$ be a regular UCL theory. An interpretation $I$ is causally explained by $T$ if and only if there exists a model $I^{*}$ of the propositional formula

$$
\begin{aligned}
\bigwedge_{F \in T} \operatorname{tr}_{p}(F) \wedge & \bigwedge_{\phi \in \text { Atom }_{\mathbf{C}}(T)}\left(a_{\phi} \supset \phi\right) \wedge \bigwedge_{\phi \in \operatorname{Atom}_{\mathbf{C}}(T)}\left(a_{\phi} \supset \widehat{\phi}\right) \\
& \wedge \bigwedge_{L \in \operatorname{Loop}(T)}\left(\left.\bigwedge_{l \in L} \hat{l} \supset \neg \bigwedge_{\phi \in \operatorname{Atom}_{\mathbf{C}}(T)}\left(a_{\phi} \supset \widehat{\phi}\right)\right|_{\widehat{L} \rightarrow \perp}\right),
\end{aligned}
$$

such that $I^{*} \cap$ Lit $=I$.

When each formula in the range of $\mathbf{C}$ is a clause in the regular UCL theory $T$, comparing the above theorem with Theorem 1 in [9], it is easy to find out that formula (12) corresponds to $D R(T) \cup C L C(T)$ in Lee's Theorem.

\section{Conclusion}

We have provided translations from Turner's logic of universal causation to propositional logic and logic programming. These translations generalize the respective translations by Ferraris and Lee for McCain and Turner's causal theories. Our next step is to use these results to implement Turner's logic using SAT and ASP solvers.

It is worth mentioning here that our results in this paper can also be used to map Turner's logic to fixed-point nonmonotonic logics such as default logic [16] and Lin and Shoham's logic of GK $[13,8]$.

Acknowledgments. This work had been supported by the National Hi-Tech Project of China under grant 2008AA01Z150, the Natural Science Foundation of China under grant 60745002 and 61175057, the USTC Key Direction Project, the Fundamental Research Funds for the Central Universities, the Youth Innovation Fund of USTC, and HK RGC GRF 616909 . We thank the anonymous reviewers for their valuable comments on an earlier version of the paper. 


\section{References}

1. Drescher, C., Gebser, M., Grote, T., Kaufmann, B., König, A., Ostrowski, M., Schaub, T.: Conflict-Driven Disjunctive Answer Set Solving. In: Brewka, G., Lang, J. (eds.) Proceedings of the 11th International Conference on Principles of Knowledge Representation and Reasoning (KR-08). pp. 422-432. AAAI Press, Menlo Park, CA, USA (2008)

2. Ferraris, P.: A logic program characterization of causal theories. In: Proceedings of the 20th International Joint Conference on Artificial Intelligence (IJCAI-07). pp. 366-371 (2007)

3. Gelfond, M., Lifschitz, V.: Classical negation in logic programs and disjunctive databases. New Generation Comput. 9(3/4), 365-386 (1991)

4. Gelfond, M., Lifschitz, V., Przymusińska, H., Truszczyński, M.: Disjunctive Defaults. In: Allen, J.F., Fikes, R., Sandewall, E. (eds.) Proceedings of the 2nd International Conference on Principles of Knowledge Representation and Reasoning (KR-91). pp. 230-237. Morgan Kaufmann, San Fransisco, CA, USA (1991)

5. Giunchiglia, E., Lee, J., Lifschitz, V., McCain, N., Turner, H.: Nonmonotonic causal theories. Artificial Intelligence 153(1-2), 49-104 (2004)

6. Giunchiglia, E., Lierler, Y., Maratea, M.: Answer Set Programming Based on Propositional Satisfiability. J. Autom. Reasoning 36(4), 345-377 (2006)

7. Janhunen, T., Niemelä, I.: GnT - A Solver for Disjunctive Logic Programs. In: Lifschitz, V., Niemelä, I. (eds.) Proceedings of the 7th International Conference on Logic Programming and Nonmonotonic Reasoning (LPNMR-04). pp. 331-335. Springer-Verlag, Berlin, Heidelberg (2004), INAI 2923

8. Ji, J., Lin, F.: From Turner's Logic of Universal Causation to the Logic of GK. In: Erdem, E., Lee, J., Lierler, Y., Pearce, D. (eds.) Correct Reasoning. Lecture Notes in Computer Science, vol. 7265, pp. 380-385. Springer (2012)

9. Lee, J.: Nondefinite vs. definite causal theories. In: Proceedings of the 7th International Conference on Logic Programming and Nonmonotonic Reasoning (LPNMR04). pp. 141-153 (2004)

10. Leone, N., Pfeifer, G., Faber, W., Eiter, T., Gottlob, G., Perri, S., Scarcello, F.: The DLV system for knowledge representation and reasoning. ACM Transactions on Computational Logic 7(3), 499-562 (2006)

11. Lifschitz, V.: Action languages, answer sets and planning. In: The Logic Programming Paradigm: a 25-Year Perspective, pp. 357-373. Springer Verlag (1999)

12. Lifschitz, V., Tang, L.R., Turner, H.: Nested expressions in logic programs. Annals of Mathematics and Artificial Intelligence 25(3-4), 369-389 (1999)

13. Lin, F., Shoham, Y.: A logic of knowledge and justified assumptions. Artificial Intelligence 57(2-3), 271-289 (1992)

14. Lin, F., Reiter, R.: Forget it. In: Working Notes of AAAI Fall Symposium on Relevance. pp. 154-159 (1994)

15. McCain, N., Turner, H.: Causal theories of action and change. In: Proceedings of the 14th National Conference on Artificial Intelligence (AAAI-97). pp. 460-465 (1997)

16. Reiter, R.: A logic for default reasoning. Artificial Intelligence 13, 81-132 (1980)

17. Turner, H.: Logic of universal causation. Artificial Intelligence 113(1), 87-123 (1999) 